\title{
The Impact of Frailty on Treatment for Overactive Bladder in Older Adults
}

\author{
Anne M. Suskind ${ }^{1}$, Casey Kowalik², Kathryn Quanstrom ${ }^{1}$, John Boscardin ${ }^{3}$, Shoujun \\ Zhao $^{1}$, W. Stuart Reynolds ${ }^{4}$, Kavita Mishra ${ }^{5}$, Emily Finlayson ${ }^{6}$ \\ ${ }^{1}$ University of California, San Francisco, Department of Urology \\ 2University of Kansas Medical Center, Department of Urology \\ ${ }^{3}$ Uinversity of California, San Francisco, Department of Biostatistics \\ ${ }^{4}$ Vanderbilt University, Department of Urology \\ ${ }^{5}$ Standford University, Department of Obstetrics and Gynecology \\ GUniversity of California, San Francisco, Department of Surgery
}

\section{Abstract}

Aims: To examine the impact of frailty on treatment outcomes for overactive bladder (OAB) in older adults starting pharmacotherapy, onabotulinumtoximA, and sacral neuromodulation.

Methods: This is a prospective study of men and women age $\geq 60$ years starting pharmacotherapy, onabotulinumtoxinA, or sacral neuromodulation. Subjects were administered questionnaires at baseline and again at 1- and 3-months. Frailty was assessed at baseline using the timed up and go test (TUGT), whereby a TUGT time of $\geq 12$ seconds was considered to be slow, or frail. Response to treatment was assessed using the overactive bladder symptom score (OABSS) and the OAB-q SF (both Bother and HRQOL subscales). Information on side effects/adverse events was also collected. Mixed effects linear modeling was used to model changes in outcomes over time both within and between groups.

Results: A total of 45 subjects enrolled in the study, $40 \%(\mathrm{~N}=18)$ of whom had a TUGT $\geq 12$ seconds. Both TUGT groups demonstrated improvement in OAB symptoms over time and there were no statistically significant differences in these responses per group (all p-values $>0.05$ ). Similar trends were found for both OAB-q SF Bother and OAB-q SF HRQOL questionnaire responses. Side effects and adverse events were not significantly different between groups (all p's $>0.05)$.

Conclusions: Adults $\geq 60$ years of age starting second- and third-line treatments for OAB, regardless of TUGT time, demonstrated improvement in OAB symptoms at 3 months. These findings suggest that frail older adults may receive comparable benefit and similar rates of side effects compared to less frail older individuals. 


\section{Introduction}

Frailty is a measure of physiologic vulnerability that manifests as increased susceptibility to adverse events such as falls, disability, loss of independence, and death. ${ }^{1,2}$ Among older individuals undergoing surgery, frailty has been linked to increased risk of postoperative complications and prolonged periods of recovery. ${ }^{3-9}$ Several validated measures that can be used to define frailty -- a complex physiologic state. Studies in surgical populations have identified slow walking speed, measured by a Timed Up and Go Test (TUGT), as a sensitive, specific, and efficient measure of frailty that has a strong independent correlation with poor surgical outcomes..$^{10,11}$

The impact of frailty on treatment outcomes for older men and women with overactive bladder (OAB), however, is poorly understood. While OAB is very common among older adults and is also known to be associated with a frail phenotype, ${ }^{12}$ most clinical trials either exclude older adults altogether or only include the healthiest of older individuals, making results from these studies poorly reflective of outcomes among real world frail older individuals. This paucity of data is problematic, as outcomes related to second- and thirdline OAB treatments are largely unknown in this population. This lack of knowledge may lead providers to either unnecessarily avoid these therapies due to theoretical concerns for reduced efficacy and/or increased side effects or, on the contrary, could lead providers to indiscriminately apply these therapies to all patients, regardless of frailty status.

In order to address these knowledge gaps and optimize treatment strategies for frail older adults with $\mathrm{OAB}$, we designed a prospective, questionnaire-based pilot study of frail and non-frail adults ages $₫ 60$ years starting pharmacotherapy, onabotuliniumtoxinA, and sacral neuromodulation. Findings from this study can be operationalized in clinical practice to inform decision-making and treatment selection among older patients (both frail and not frail) with OAB. Furthermore, a better understanding of the role of frailty in this population is novel and essential to serving the rapidly growing older population.

\section{Materials and Methods}

\section{Subjects and study design}

This is a questionnaire-based study of men and women ages $\geq 60$ years who were starting treatments for $\mathrm{OAB}$ including pharmacotherapy (both antimuscarinics and $\beta 3$-agonists), onabotulinumtoxinA, and sacral neuromodulation from 2015 to 2018. OAB treatments for study participants were selected based on routine clinical practice. Study participants were recruited from urology and urogynecology practices two different academinc institutions. All participants signed informed consent and the study was approved by the Institutional Review Boards at both study sites. Subjects were excluded if they spoke a primary language other than English or were unable to self-consent. Subjects who required help completing the questionnaires were included in the study if this help was readily available from a family member or caregiver.

Subjects completed assessments and questionnaires at baseline (marking the commencement of OAB therapy), and follow-up questionnaires were performed at 1-month and 3-months 
after starting treatment. Due to difficulty with recruitment and retainment of subjects, partway through the study, subjects were offered gift cards in the sum of $\$ 15$ for the completion of each questionnaire.

\section{Outcomes}

The primary outcomes for this study were assessment of OAB symptoms according to questionnaires at baseline, 1-month and 3-months using the Overactive Bladder Symptom Score (OABSS) and the Overactive Bladder Questionnaire Short Form (OAB-q SF), both Bother and Health Related Quality of Life (HRQOL) subscales.

The OABSS is a validated tool that consists of four questions addressing daytime frequency, nighttime frequency, urgency and urgency incontinence. Scores range from a minimum of 0 to a maximum of $15^{13}$ depending on degree of $\mathrm{OAB}$ severity, with higher scores representing increased symptom severity.

The OAB-q SF is a validated patient-reported outcome measure that captures the spectrum of OAB symptoms including health-related quality of life (HRQOL) and bother. The short form is an abbreviated version of the OAB-q that was developed using psychometric principles. ${ }^{14}$ The OAB-q SF is more parsimonious than the OAB-q and includes 19 questions in two scales consisting of a 13-item HRQOL scale (OAB-q SF HRQOL) and a 6item symptom Bother scale (OAB-q SF Bother). The HRQOL score ranges from 13 to 78 and the Bother scores range from 6 to 36, with higher scores representing more severe symptoms.

The secondary outcomes included side effects and adverse events measured at 1 and 3 months. These were self-reported via questionnaires at both time points and included the following: headache, dry mouth, constipation, urinary tract infection (UTI), nausea, urinary retention, fatigue, confusion, and pain.

\section{Covariates}

The baseline assessment included a post void residual (PVR) measured by bladder ultrasound or catheterization, an animal fluency test, and a TUGT. The animal fluency test is a type of verbal fluency measure whereby the subject is asked to name as many animals that they can think of in 60 seconds. This brief measure has been shown to effectively distinguish normal controls and persons with mild Alzheimer's Dementia, ${ }^{15}$ cognitive impairment without dementia, ${ }^{16}$ and mild cognitive impairment. ${ }^{17}$ Individuals who cannot name 15 or more animals during 60 seconds may have 20 times higher likelihood of dementia compared to individuals who can name 15 or more animals. ${ }^{16}$

The TUGT was administered to all subjects as our measure of frailty, ${ }^{11}$ whereby each individual was instructed to stand up from a seated position in a chair, walk 10 feet at a normal pace (individuals can use a walker, cane, or other walking device if needed), turn around, walk back to the chair, then sit back down. This task requires assimilation of several elements including understanding and following instructions, the use of core strength to transfer from a seated to a standing position (and vice versa), walking speed and balance. The time required to complete this task is recorded in seconds. A time of $<12$ seconds is 
considered "fast", correlating to "not frail", and a time of $\geq 12$ seconds is considered

"impaired", correlating to "frail". ${ }^{18}$

The baseline questionnaire included information on prior OAB treatments including pelvic floor physical therapy, pharmacotherapy (and type), onabotulinumtoxinA, sacral neuromodulation and percutaneous tibial nerve stimulation (PTNS). Activities of daily living (ADLs) and instrumental activities of daily living (IADLs) were obtained via the Katz Index of Independence in ADL (Katz ADL) and the Lawton Instrumental Activities of Daily Living Scale (Lawton IADL). The Katz ADL is the gold-standard measure of functional status, or of an individual's ability to perform ADLs independently. This index asks questions pertaining to bathing, toileting, transferring, continence and feeding. Scores range from 0 (severe functional impairment) to 6 (full function). Katz ADL scores provide useful information about prognosis and can be used to monitor health and illness in older individuals. ${ }^{19}$ The Lawton IADL items are considered more complex than those items in the Katz ADL and consist of 8 domains of function: ability to use the telephone, shopping, food preparation, housekeeping, laundry, mode of transportation, responsibility for own medication, and ability to handle finances. Scores range from 0 (low function, dependent) to 8 (high function, independent). ${ }^{20}$

Additional information on self-rated health (excellent, very good, good, fair, poor), race/ ethnicity, highest degree or level of education completed, and total annual household income was solicited on the baseline questionnaire.

\section{Statistical analysis}

Summary characteristics and side effects were compared between individuals based on TUGT times ( $<12$ seconds versus $\geq 12$ seconds) and were presented as averages with standard deviations or as numbers with percentages, where appropriate. Groups were compared using Mann-Whitney and chi-square $t$ tests, where appropriate, with two-sided $P$ values of $<0.05$ being considered significant.

OAB questionnaire scores (OABSS, OAB-q SF HRQOL and Bother subscales) were modeled over time (at baseline, 1-month, and 3-months) adjusting for age and neurogenic bladder status. Results were reported as LS Means with $95 \%$ confidence intervals. P values were calculated for each TUGT subgroup separately over time and for differences between the TUGT groups over time. Due to missing data on some subjects over time, we performed a sensitivity analysis for our main outcome (each OAB questionnaire), whereby only the patients with complete data at all three time points were modeled separately over time. Analyses were performed using SAS 9.3 software.

\section{Results}

The flow diagram, according to CONSORT statement, depicting enrollment in the study is shown in figure 1. Sixty-four individuals were originally enrolled in the study across the two sites, 45 individuals completed the baseline questionnaire, 38 completed the 1-month questionnaire and 33 completed the 3 -month questionnaire. Of note, there were 4 study 
participants who completed the baseline and 3-month questionnaires but not the 1-month questionnaire.

Baseline characteristics of the study cohort are shown in Table 1. Among the 45 study participants, 27 had a TUGT $<12$ seconds and 18 had a TUGT $\geq 12$ seconds. The two groups were similar in most baseline demographic characteristics with the exception of neurogenic bladder status, whereby $7.4 \%$ of individuals with a TUGT $<12$ seconds and $33.3 \%$ of individuals with a TUGT $\geq 12$ seconds had a diagnosis of neurogenic bladder. Function was slightly better in the TUGT $<12$ seconds group compared to the TUGT $\geq 12$ seconds group, as measured by Katz ADLs ( $5.5 \pm 0.5$ compared to $5.0 \pm 0.6)$ and Lawton IADLs ( $7.6 \pm 1.2$ compared to $6.6 \pm 1.5)$. Self-rated general health almost reached statistical significance with the TUGT $\geq 12$ second group leaning towards slightly worse general health $(\mathrm{p}=0.05)$. Age, gender, BMI, race/ethnicity, education, total household income, number of medications, PVR, animal fluency test scores, and previous treatments tried did not statistically differ between groups.

Table 2 shows the type of OAB treatment selected (as part of usual care) during the study. There were no differences in treatment selection by TUGT group and nearly half of each group received a medication, $48.1 \%$ for the TUGT $<12$ seconds group and $50.0 \%$ for the TUGT $\geq 12$ seconds group. The only statistically significant difference between groups was that a higher percentage of individuals in the TUGT $\geq 12$ seconds group received mirabegron compared to the TUGT $<12$ seconds group ( $16.7 \%$ compared to $0 \%$, respectively).

Figure 2 illustrates the longitudinal LS means (with 95\% CI's) for each OAB questionnaire (OABSS, OAB-q SF HRQOL and OAB-q SF Bother) over the 3-month study period, adjusted for age and neurogenic bladder status. Each figure shows a similar trend of statistically significant improvement in OAB symptoms with treatment over time (all $\mathrm{p}$ values $<0.05$ ) and statistically insignificant differences in trends between each group over time (all $\mathrm{p}$ values $\geq 0.05$ ).

Due to missing data from some of the study participants at different time points, we conducted a sensitivity analysis whereby we compared modeled trajectories over time between the 29 subjects that had complete data at all 3 time points with the entire study cohort of 45 subjects. The models for the 29 subjects with complete data showed similar trends to the models presented in Figure 2 on the entire study cohort (data not shown).

Table 3 presents results pertaining to treatment side effects. Overall, $73.7 \%$ of study participants experienced side effects at 1 -month and $81.8 \%$ at 3 -months. There were no statistically significant differences in side effects between TUGT groups either overall or by individual side effect (all $\mathrm{p}$ values $>0.05$ ).

\section{Discussion}

After starting pharmacotherapy, onabotulinumtoxin $\mathrm{A}$ or sacral neuromodulation for $\mathrm{OAB}$ treatment, both frail and non-frail older adults demonstrated statistically significant improvement in $\mathrm{OAB}$ symptoms, as measured by three OAB questionnaires (OABSS, OABq SF HRQOL and OAB-q SF Bother) over a period of three months. These improvements 
did not statistically differ between frailty groups and the two groups demonstrated comparable rates of side effects.

Our study is unique in that it focuses on frail older adults with OAB. Limited studies assessing $\mathrm{OAB}$ treatment outcomes among frail older adults exist. Most of these studies are limited to pharmacotherapy, which approximately half of our study participants received. Four studies looked at oxybutynin use among frail older populations, most of which demonstrated limited efficacy. Of note, only three of our study participants received this particular medication. One of these studies evaluated female nursing home residents with mild to severe cognitive impairment and randomized residents to oxybutynin $5 \mathrm{mg}$ daily or placebo over 4 weeks. This study found no significant improvement in urinary incontinence, urinary urgency or the achievement of dryness among the treatment group and there were no differences in rates of delirium between the treatment and placebo groups. ${ }^{21,22} \mathrm{~A}$ study in frail older community-dwelling individuals randomized subjects to oxybutynin or placebo and found that oxybutynin was superior to placebo in reducing daytime frequency and increasing subjective benefit; however, there was no difference in incontinence episodes. The frequency of side effects was similar between both groups at $50 \% .{ }^{23} \mathrm{~A}$ small study looking at 24 incontinent institutionalized older adults randomized to oxybutynin $5 \mathrm{mg}$ twice daily or placebo found no clinically significant differences in incontinence (as measured by a bedside electronic monitor) over 8 days. ${ }^{24}$ Finally, a randomized study looking at the addition of oxybutynin to prompted voiding plus placebo among nursing home residents with urinary incontinence demonstrated statistically significant, but not clinically meaningful, reductions in the frequency of urinary incontinence between groups. ${ }^{25}$

While the literature on oxybutynin demonstrates limited efficacy among frail older adults, the use of other antimuscarincs may be more promising in this population. One randomized study evaluated the use of fesoterodine versus placebo among a cohort of medically complex, vulnerable, community-dwelling men and women ages $\Varangle 65$ years of age over a period of 12 weeks. Subjects in the fesoterodine arm demonstrated statistically significant improvement in $\mathrm{OAB}$ symptoms compared to placebo and similar rates of adverse events compared to younger populations, concluding that fesoterodine was both efficacious and well-tolerated in this medically complex population. ${ }^{26}$ This study not only included a frailer population, but it also demonstrated better outcomes compared to the literature on oxybutynin.

Mirabegron, a $\beta 3$-agonist, is thought to have great promise for use among frail older adults due to a lower incidence of central nervous system side effects compared to antimuscarinics, 27 therefore, it is not surprising that more frail subjects in our study received mirabegron compared to non-frail older adults ( $\mathrm{p}=0.03$ ). One study in the literature looked at older adults ages $\geq 65$ and $\geq 75$ and found similar efficacy and tolerability of the medication between age groups over a period of 12 weeks. While this study is promising, it did not incorporate frailty and existing data in the literature on frail older adults taking mirabegron are lacking. ${ }^{27}$

Approximately one quarter of our study participants in each group received onabotulinumtoxinA injections. The use of onabotulinumtoxinA injections was evaluated in 
one study among older frail, older non-frail, and younger (age <65) adults with idiopathic refractory overactive bladder symptoms. All subjects received 100 units of onabotulinumtoxinA and were defined as frail if they met 3 or more of the following criteria: unintentional weight loss, self-reported exhaustion, weakness, slow walking speed or low physical activity. Elevated post void residual $(>150 \mathrm{~mL})$ was significantly higher in the frail group compared to the older non-frail and younger groups $(60.7 \%$ vs $39.7 \%$ and $35.7 \%$, respectively, $\mathrm{p}=0.018$ ). Urinary retention was more common in the older frail group, followed by the older non-frail and younger age groups $(11.5 \%, 6.3 \%$, and $2.4 \%$, respectively, $\mathrm{p}=0.203$ ), and cumulative success rate was significantly lower in the frail older group compared to the other two groups $(\mathrm{p}=0.009) .{ }^{28}$

Unfortunately, no studies inclusive of the frail older population undergoing treatment with sacral neuromodulation for $\mathrm{OAB}$ currently exist. A secondary analysis of the Refractory Overactive Bladder: Sacral Neuromodulation vs Botulinum Toxin Assessment (ROSETTA) study did not evaluate frailty, but did compare women $<65$ and $\geq 65$ years randomized to sacral neuromodulation or onabotulinumtoxinA. The study found that women in the younger age group experienced greater rates of continence and symptom improvement with fewer urinary tract infections; however, women in both age groups demonstrated benefits in reduction in urgency urinary incontinence episodes with similar rates of adverse events and improvement in quality of life. ${ }^{29}$ While this study reported decreased efficacy in older adults compared to younger adults, older adults still demonstrated symptom improvement with both types of third-line therapies.

Our study should be interpreted with certain limitations in mind. First, this study is limited by risk of type 2 error due to small numbers $(\mathrm{N}=45)$. For this reason, we were unable to perform certain analyses, such as stratification by treatment type. While this is an unfortunate limitation of our study, it is important to keep in mind that this is a pilot study and we acknowledge the need for future larger studies in order to confirm and expand on these findings. Despite this limitation, we strongly feel that these findings serve as an important addition to the literature, which is almost entirely lacking in its description of frail older adults undergoing common OAB treatments. Second, there were some missing data whereby not all study participants completed all three questionnaires. For this reason, we performed a sensitivity analysis and found no difference in our primary outcome (OAB response measured by all three questionnaires) between subjects who completed all questionnaires and those who did not. Therefore, we do not believe that this limited our study or our study conclusions in any way.

\section{Conclusions}

Second- and third-line OAB treatments in the form of pharmacotherapy, onabotulinumtoxinA and sacral neuromodulation demonstrated symptom improvement in both frail and non-frail older adults over a period of 3 months. Additionally, there were no statistically significant differences in treatment outcomes between frail and non-frail groups over time and rates of side effects were also similar between groups. This pilot study is among the first to evaluate the use of $\mathrm{OAB}$ treatments in frail older adults and suggests that 
both second- and third-line OAB treatments may be considered for use in appropriate frail older adults.

\section{Funding:}

Society of Urodynamics, Female Pelvic Medicine and Urogenital Reconstruction (SUFU) and Pfizer Grant for the Study of Overactive Bladder and Urgency Urinary Incontinence; 2015

\section{References}

1. Buchner DM, Wagner EH. Preventing frail health. Clinics in geriatric medicine. 1992;8(1):1-17.

2. Fried LP, Tangen CM, Walston J, et al. Frailty in older adults: evidence for a phenotype. The journals of gerontology Series A, Biological sciences and medical sciences. 2001;56(3):M146-156.

3. Makary MA, Segev DL, Pronovost PJ, et al. Frailty as a predictor of surgical outcomes in older patients. Journal of the American College of Surgeons. 2010;210(6):901-908. [PubMed: 20510798]

4. Green P, Arnold SV, Cohen DJ, et al. Relation of Frailty to Outcomes After Transcatheter Aortic Valve Replacement (from the PARTNER Trial). The American journal of cardiology. 2015.

5. Partridge JS, Fuller M, Harari D, Taylor PR, Martin FC, Dhesi JK. Frailty and poor functional status are common in arterial vascular surgical patients and affect postoperative outcomes. Int J Surg. 2015;18:57-63. [PubMed: 25907322]

6. Ambler GK, Brooks DE, Al Zuhir N, et al. Effect of frailty on short- and mid-term outcomes in vascular surgical patients. The British journal of surgery. 2015;102(6):638-645. [PubMed: 25764503]

7. Chen CC, Lin MT, Liang JT, Chen CM, Yen CJ, Huang GH. Pre-surgical Geriatric Syndromes, Frailty, and Risks for Postoperative Delirium in Older Patients Undergoing Gastrointestinal Surgery: Prevalence and Red Flags. Journal of gastrointestinal surgery : official journal of the Society for Surgery of the Alimentary Tract. 2015;19(5):927-934. [PubMed: 25650167]

8. Suskind AM, Jin C, Cooperberg MR, et al. Preoperative Frailty Is Associated With Discharge to Skilled or Assisted Living Facilities After Urologic Procedures of Varying Complexity. Urology. 2016.

9. Suskind AM, Walter LC, Jin C, et al. Impact of frailty on complications in patients undergoing common urological procedures: a study from the American College of Surgeons National Surgical Quality Improvement database. BJU international. 2016;117(5):836-842. [PubMed: 26691588]

10. Robinson TN, Wu DS, Sauaia A, et al. Slower walking speed forecasts increased postoperative morbidity and 1-year mortality across surgical specialties. Annals of surgery. 2013;258(4):582588; discussion 588-590. [PubMed: 23979272]

11. Savva GM, Donoghue OA, Horgan F, O'Regan C, Cronin H, Kenny RA. Using timed up-and-go to identify frail members of the older population. The journals of gerontology Series A, Biological sciences and medical sciences. 2013;68(4):441-446.

12. Suskind AM, Quanstrom K, Zhao S, et al. Overactive Bladder Is Strongly Associated With Frailty in Older Individuals. Urology. 2017;106:26-31. [PubMed: 28502833]

13. Homma Y, Yoshida M, Seki N, et al. Symptom assessment tool for overactive bladder syndrome-overactive bladder symptom score. Urology. 2006;68(2):318-323. [PubMed: 16904444]

14. Coyne KS, Thompson CL, Lai JS, Sexton CC. An overactive bladder symptom and health-related quality of life short-form: validation of the OAB-q SF. Neurourology and urodynamics. 2015;34(3):255-263. [PubMed: 25783168]

15. Monsch AU, Bondi MW, Butters N, Salmon DP, Katzman R, Thal LJ. Comparisons of verbal fluency tasks in the detection of dementia of the Alzheimer type. Archives of neurology. 1992;49(12):1253-1258. [PubMed: 1449404]

16. Canning SJ, Leach L, Stuss D, Ngo L, Black SE. Diagnostic utility of abbreviated fluency measures in Alzheimer disease and vascular dementia. Neurology. 2004;62(4):556-562. [PubMed: 14981170] 
17. Woodard JL, Dorsett ESW, Cooper JG, Hermann BP, Sager MA. Development of a Brief Cognitive Screen for Mild Cognitive Impairment and Neurocognitive Disorder. Neuropsychol Dev Cogn B Aging Neuropsychol Cogn. 2005;12(4):299-315. [PubMed: 28486831]

18. Bischoff HA, Stahelin HB, Monsch AU, et al. Identifying a cut-off point for normal mobility: a comparison of the timed 'up and go' test in community-dwelling and institutionalised elderly women. Age and ageing. 2003;32(3):315-320. [PubMed: 12720619]

19. Katz S Assessing self-maintenance: activities of daily living, mobility, and instrumental activities of daily living. Journal of the American Geriatrics Society. 1983;31(12):721-727. [PubMed: 6418786]

20. Lawton MP, Brody EM. Assessment of older people: self-maintaining and instrumental activities of daily living. The Gerontologist. 1969;9(3):179-186. [PubMed: 5349366]

21. Lackner TE, Wyman JF, McCarthy TC, Monigold M, Davey C. Efficacy of oral extended-release oxybutynin in cognitively impaired older nursing home residents with urge urinary incontinence: a randomized placebo-controlled trial. Journal of the American Medical Directors Association. 2011;12(9):639-647. [PubMed: 21450183]

22. Lackner TE, Wyman JF, McCarthy TC, Monigold M, Davey C. Randomized, placebo-controlled trial of the cognitive effect, safety, and tolerability of oral extended-release oxybutynin in cognitively impaired nursing home residents with urge urinary incontinence. Journal of the American Geriatrics Society. 2008;56(5):862-870. [PubMed: 18410326]

23. Szonyi G, Collas DM, Ding YY, Malone-Lee JG. Oxybutynin with bladder retraining for detrusor instability in elderly people: a randomized controlled trial. Age and ageing. 1995;24(4):287-291. [PubMed: 7484484]

24. Zorzitto ML, Holliday PJ, Jewett MA, Herschorn S, Fernie GR. Oxybutynin chloride for geriatric urinary dysfunction: a double-blind placebo-controlled study. Age and ageing. 1989;18(3):195200. [PubMed: 2675580]

25. Ouslander JG, Schnelle JF, Uman G, et al. Does oxybutynin add to the effectiveness of prompted voiding for urinary incontinence among nursing home residents? A placebo-controlled trial. Journal of the American Geriatrics Society. 1995;43(6):610-617. [PubMed: 7775717]

26. Dubeau CE, Kraus SR, Griebling TL, et al. Effect of fesoterodine in vulnerable elderly subjects with urgency incontinence: a double-blind, placebo controlled trial. The Journal of urology. 2014;191(2):395-404. [PubMed: 23973522]

27. Wagg A, Cardozo L, Nitti VW, et al. The efficacy and tolerability of the beta3-adrenoceptor agonist mirabegron for the treatment of symptoms of overactive bladder in older patients. Age and ageing. 2014;43(5):666-675. [PubMed: 24610862]

28. Liao CH, Kuo HC. Increased risk of large post-void residual urine and decreased long-term success rate after intravesical onabotulinumtoxinA injection for refractory idiopathic detrusor overactivity. The Journal of urology. 2013;189(5):1804-1810. [PubMed: 23178902]

29. Komesu YM, Amundsen CL, Richter HE, et al. Refractory urgency urinary incontinence treatment in women: impact of age on outcomes and complications. American journal of obstetrics and gynecology. 2018;218(1):111 e111-111 e119. [PubMed: 29031894] 


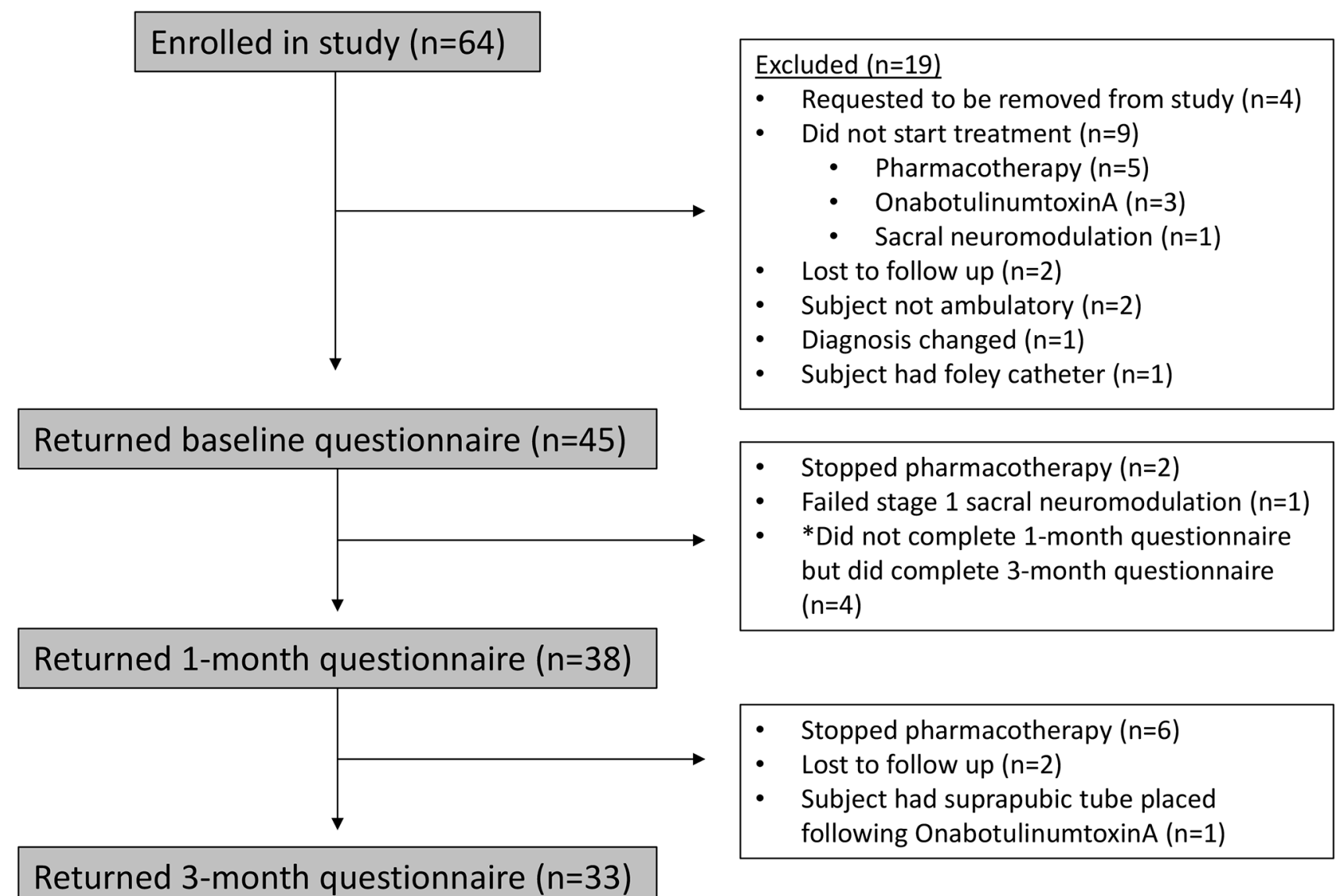

Figure 1:

CONSORT diagram depicting study enrollment. 
A.

- - - . TUGT $\geqq 12$



B.

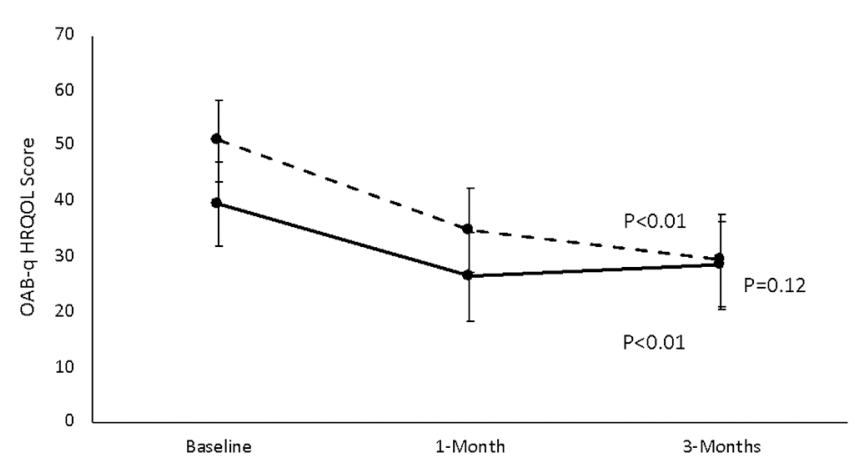

C.

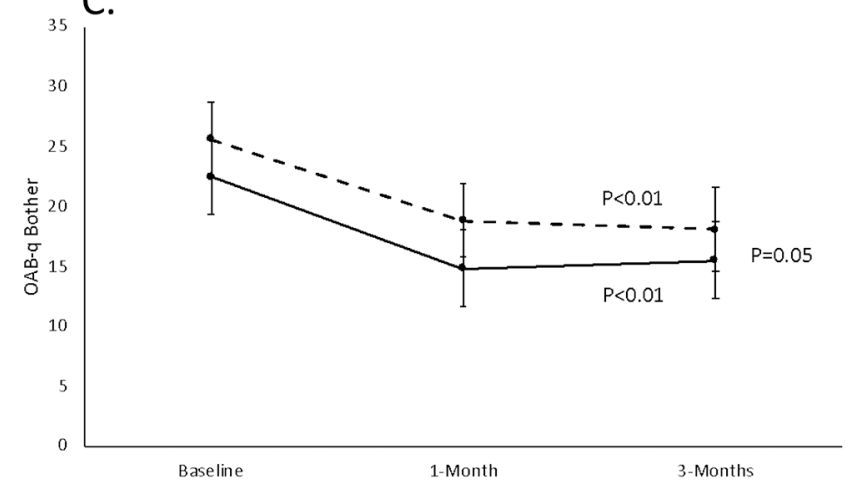

Figure 2.

Questionnaire-based treatment responses to OAB treatments stratified by TUGT $<12$ and TUGT $\geqq 12$. Panel A is the OABSS, B is OAB-q HRQOL and C is OAB-q Bother. All figures are adjusted for age and neurogenic bladder. 


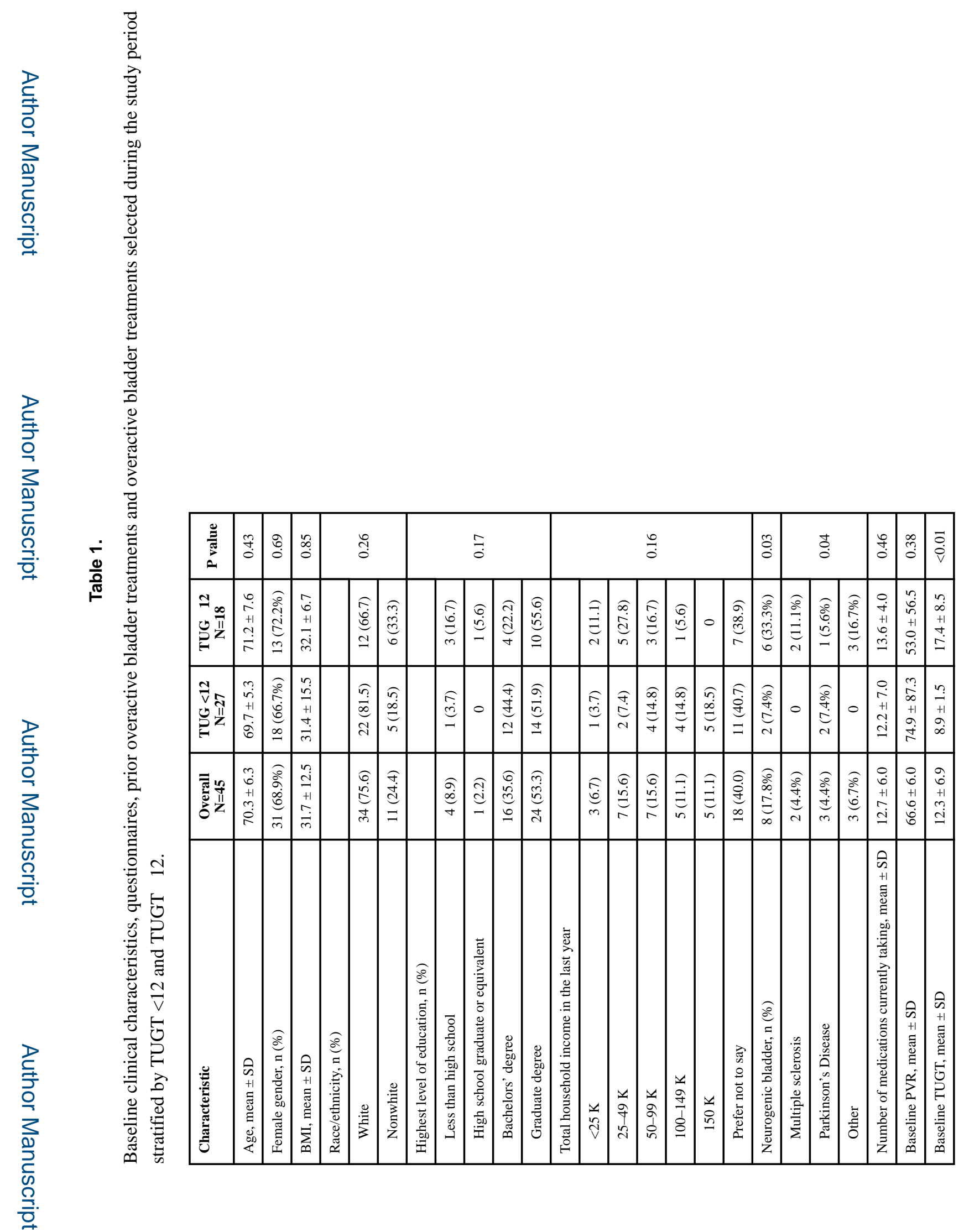

Neurourol Urodyn. Author manuscript; available in PMC 2019 September 01. 


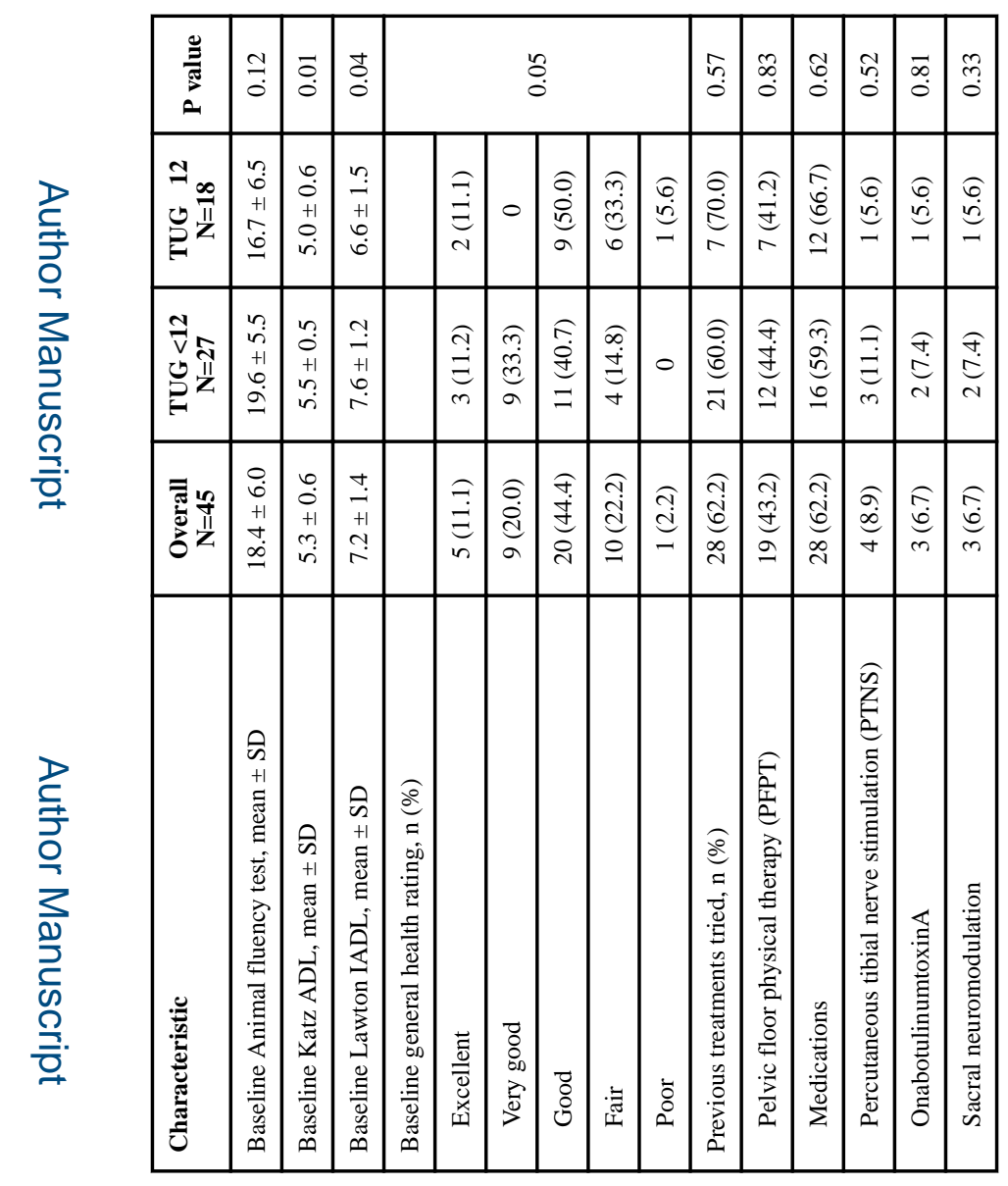

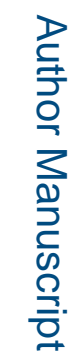

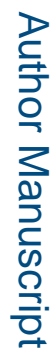

Neurourol Urodyn. Author manuscript; available in PMC 2019 September 01. 

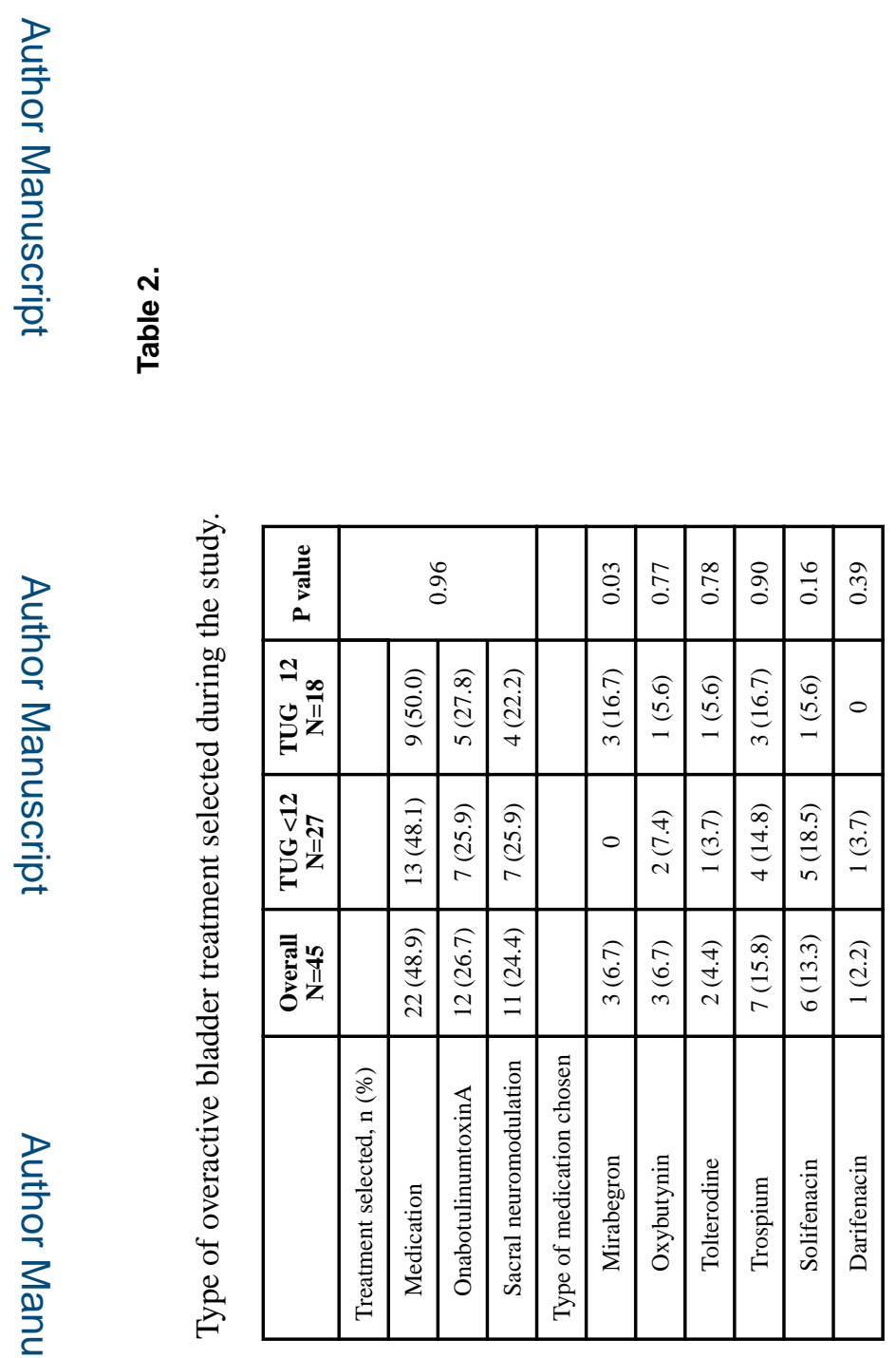

Neurourol Urodyn. Author manuscript; available in PMC 2019 September 01. 


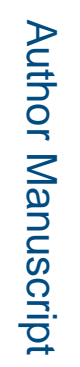

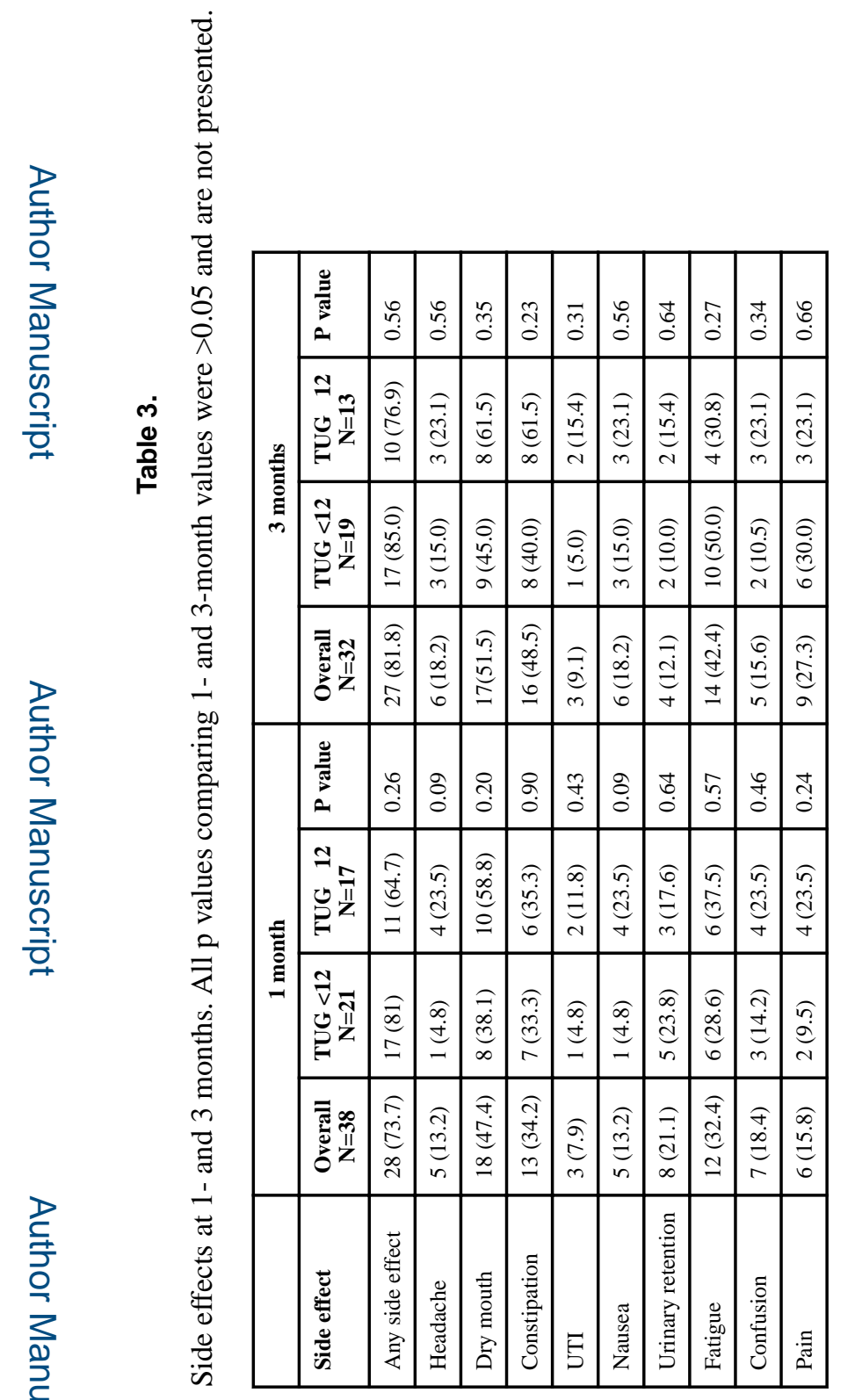

Neurourol Urodyn. Author manuscript; available in PMC 2019 September 01. 